\title{
ADAPTIVE BACKSTEPPING CONTROLLER AND SYNCHRONIZER DESIGN FOR ARNEODO CHAOTIC SySTEM WiTH UNKNOWN PARAMETERS
}

\author{
Sundarapandian Vaidyanathan ${ }^{1}$ \\ ${ }^{1}$ Research and Development Centre, Vel Tech Dr. RR \& Dr. SR Technical University \\ Avadi, Chennai-600 062, Tamil Nadu, INDIA \\ sundarvtu@gmail.com
}

\begin{abstract}
In this paper, we apply backstepping control method to derive new results for the adaptive controller and synchronizer design for the Arneodo chaotic system (1980), when the system parameters are unknown. First, we design an adaptive backstepping controller to stabilize the Arneodo system to its unstable equilibrium at the origin. Next, we design an adaptive backstepping controller to achieve global chaos synchronization of the identical Arneodo chaotic systems with unknown parameters. MATLAB simulations have been detailed to illustrate the proposed adaptive backstepping controller and synchronizer design for Arneodo chaotic system with unknown parameters.
\end{abstract}

\section{KEYWORDS}

Backstepping Control, Adaptive Control, Adaptive Synchronization, Chaos, Arneodo System.

\section{INTRODUCTION}

Chaos theory is the qualitative study of unstable aperiodic behaviour in deterministic nonlinear dynamical systems. A chaotic system is popularly known as nonlinear dynamical system, which is very sensitive to initial conditions. In 1963, Lorenz discovered that a very small difference in the initial conditions led to large changes in his deterministic weather model [1]. Other classical 3-dimensional chaotic systems include Rössler system [2], Newton-Leipnik system [3], Chen system [4] and Lü system [5].

The problem of controlling a chaotic system aims to find a state feedback control law to stabilize the chaotic system around its unstable equilibrium points [6-7]. We use active control method [89], when the system parameters are known and we use adaptive control method [10-12], when the system parameters are unknown.

The problem of synchronizing chaotic systems aims to find a state feedback control law to synchronize a pair of coupled chaotic systems known as master-slave systems or drive-response systems.

The seminal paper on synchronization of chaotic systems was published by Pecora and Carroll in 1990 [13]. Afterwards, chaos synchronization has found applications in many fields such as physics [14-15], chemistry [16], ecology [17], biology [18], cardiology [19], neural networks [20], robotics [21-22], secure communications [23-24].

DOI : $10.5121 /$ ijcsit.2012.4611 
Some commonly used methods for addressing chaos synchronization problem are active control method [25-30], adaptive control method [31-35], sampled-data feedback method [36], timedelay feedback method [37], sliding mode control method [38-44], backstepping control method [45-48], etc.

In this paper, we derive new results for the adaptive backstepping controller and adaptive backstepping synchronizer for the Arneodo chaotic system ([47], 1980) with unknown parameters. The stability results have been established using Lyapunov stability theory. MATLAB simulations have been detailed for the adaptive controllers derived in the paper.

\section{SYSTEM DESCRIPTION}

The Arneodo system ([47], 1980) is given by the 3-dimensional dynamics

$$
\begin{aligned}
& \dot{x}_{1}=x_{2} \\
& \dot{x}_{2}=x_{3} \\
& \dot{x}_{3}=a x_{1}-b x_{2}-x_{3}-x_{1}^{2}
\end{aligned}
$$

where $x_{1}, x_{2}, x_{3}$ are the states and $a, b$ are constant, positive parameters of the system. Arneodo system (1) undergoes chaotic behaviour when the parameter values are

$$
a=7.5 \text { and } b=3.8
$$

Figure 1 depicts the strange chaotic attractor of the Arneodo chaotic system.

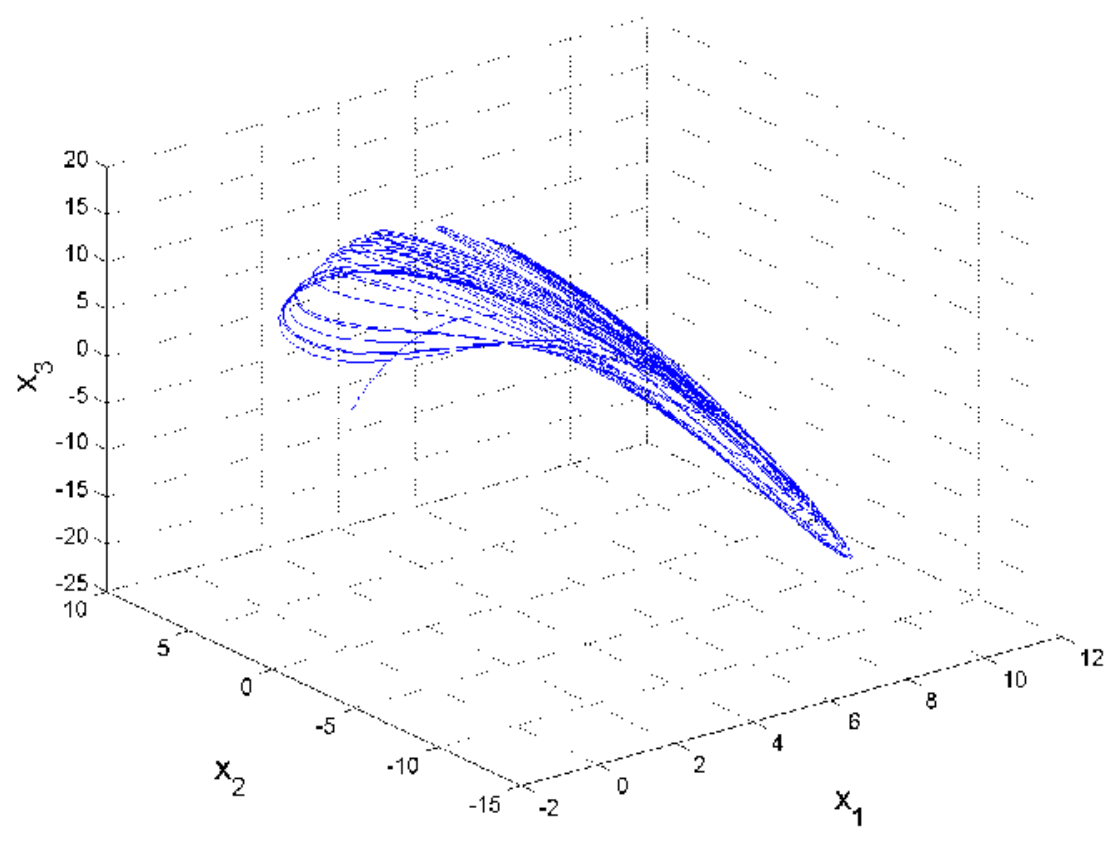

Figure 1. Strange Attractor of the Arneodo System 
When the parameter values are taken as in (2) for Arneodo system (1), the system linearization matrix at the origin is given by

$$
A=\left[\begin{array}{ccc}
0 & 1 & 0 \\
0 & 0 & 1 \\
a & -b & -1
\end{array}\right]
$$

which has the characteristic polynomial

$$
p(\lambda)=\operatorname{det}(\lambda I-A)=\lambda^{3}+\lambda^{2}+b \lambda-a
$$

Since $a, b>0$, it is immediate that the coefficients of $p(\lambda)$ are not all positive. Hence, by Routh-Hurwitz criterion, the matrix $A$ has an unstable eigenvalue. Thus, it is immediate that the Arneodo system (1) is unstable at the origin.

\section{Adaptive Backstepping Control of the Arneodo Chaotic SYSTEM}

\subsection{Main Results}

In this section, we design an adaptive backstepping controller for globally stabilizing the Arneodo system (1980) with unknown parameters.

Thus, we consider the controlled Arneodo system

$$
\begin{aligned}
& \dot{x}_{1}=x_{2} \\
& \dot{x}_{2}=x_{3} \\
& \dot{x}_{3}=a x_{1}-b x_{2}-x_{3}-x_{1}^{2}+u
\end{aligned}
$$

where $u$ is a backstepping controller to be designed using the states $x_{1}, x_{2}, x_{3}$ and estimates $\hat{a}(t), \hat{b}(t)$ of the unknown parameters $a, b$ of the system.

The parameter estimation errors are defined by

$e_{a}(t)=a-\hat{a}(t)$
$e_{b}(t)=b-\hat{b}(t)$

Note that

$$
\begin{aligned}
& \dot{e}_{a}(t)=-\dot{\hat{a}}(t) \\
& \dot{e}_{b}(t)=-\dot{\hat{b}}(t)
\end{aligned}
$$

The main result for the adaptive backstepping controller design for the Arneodo system (3) is described by the following theorem. 
Theorem 1. The Arneodo chaotic system (3) with unknown parameters and is globally and exponentially stabilized for all values of $x(0) \in R^{3}$ by the backstepping controller

$u(t)=-(\hat{a}+3) x_{1}-(5-\hat{b}) x_{2}-2 x_{3}+x_{1}^{2}$

where $\hat{a}(t), \hat{b}(t)$ are estimates of the unknown parameters $a, b$ and the parameter update law is given by

$\dot{\hat{a}}=\left(2 x_{1}+2 x_{2}+x_{3}\right) x_{1}+k_{a} e_{a}$

$\dot{\hat{b}}=-\left(2 x_{1}+2 x_{2}+x_{3}\right) x_{2}+k_{b} e_{b}$

with gains $k_{a}, k_{b}>0$.

Proof. We establish the main result using Lyapunov stability theory [48].

First, we define a Lyapunov function candidate

$V_{1}=\frac{1}{2} z_{1}^{2}$

where $z_{1}=x_{1}$.

Differentiating $V_{1}$ along the solutions of the Arneodo system (3), we get

$\dot{V}_{1}=z_{1} \dot{z}_{1}=x_{1} x_{2}=-z_{1}^{2}+z_{1}\left(x_{1}+x_{2}\right)$

Secondly, we choose the second Lyapunov function candidate as

$V_{2}=V_{1}+\frac{1}{2} z_{2}^{2}=\frac{1}{2}\left(z_{1}^{2}+z_{2}^{2}\right)$

where $z_{2}=x_{1}+x_{2}$.

Differentiating $V_{2}$ along the solutions of the Arneodo system (3), we get

$\dot{V}_{2}=-z_{1}^{2}-z_{2}^{2}+z_{2}\left(2 x_{1}+2 x_{2}+x_{3}\right)$

Finally, for the systems (3) and (5), we consider the Lyapunov function candidate

$V=V_{2}+\frac{1}{2} z_{3}^{2}+\frac{1}{2} e_{a}^{2}+\frac{1}{2} e_{b}^{2}=\frac{1}{2}\left(z_{1}^{2}+z_{2}^{2}+z_{3}^{2}+e_{a}^{2}+e_{b}^{2}\right)$

where $z_{3}=2 x_{1}+2 x_{2}+x_{3}$. 
Differentiating $V$ along the solutions of the systems (3) and (5), we obtain

$$
\dot{V}=-z_{1}^{2}-z_{2}^{2}-z_{3}^{2}+z_{3}\left[(3+a) x_{1}+(5-b) x_{2}+2 x_{3}-x_{1}^{2}+u\right]-e_{a} \dot{\hat{a}}-e_{b} \dot{\hat{b}}
$$

Substituting the backstepping controller (6) into (13), we obtain

$$
\dot{V}=-z_{1}^{2}-z_{2}^{2}-z_{3}^{2}+z_{3}\left(e_{a} x_{1}-e_{b} x_{2}\right)-e_{a} \dot{\hat{a}}-e_{b} \dot{\hat{b}}
$$

i.e.

$$
\dot{V}=-z_{1}^{2}-z_{2}^{2}-z_{3}^{2}+e_{a}\left(z_{3} x_{1}-\dot{\hat{a}}\right)+e_{b}\left(-z_{3} x_{2}-\dot{\hat{b}}\right)
$$

Substituting the parameter update law (7) into (14), we get

$$
\dot{V}=-z_{1}^{2}-z_{2}^{2}-z_{3}^{2}-k_{a} e_{a}^{2}-k_{b} e_{b}^{2}
$$

which is a negative definite function.

Hence, by Lyapunov stability theory [48], the proof is complete.

\subsection{Numerical Results}

For numerical simulations, we have applied the fourth order Runge-Kutta method (MATLAB) with the step-size $h=10^{-8}$ to solve the Arneodo system (3) with the adaptive backstepping control law (6) and the parameter update law (7).

The parameters of the Arneodo system (3) are taken as in the chaotic case, i.e.

$$
a=7.5, \quad b=3.8
$$

For the adaptive and update laws, we take $k_{a}=6$ and $k_{b}=6$.

Suppose that the initial values of the estimated parameters are

$$
\hat{a}(0)=15, \quad \hat{b}(0)=6
$$

The initial state of the controlled Arneodo system (3) is taken as

$$
x_{1}(0)=25, \quad x_{2}(0)=-16, \quad x_{3}(0)=30
$$

When the adaptive control law (6) and the parameter update law (7) are used, the state trajectories of the controlled Arneodo system converge exponentially to the equilibrium at the origin as shown in Figure 2.

The time-history of the parameter estimates $\hat{a}(t), \hat{b}(t)$ is shown in Figure 3 . 
International Journal of Computer Science \& Information Technology (IJCSIT) Vol 4, No 6, December 2012

The time-history of the parameter estimation errors $e_{a}(t), e_{b}(t)$ is shown in Figure 4.

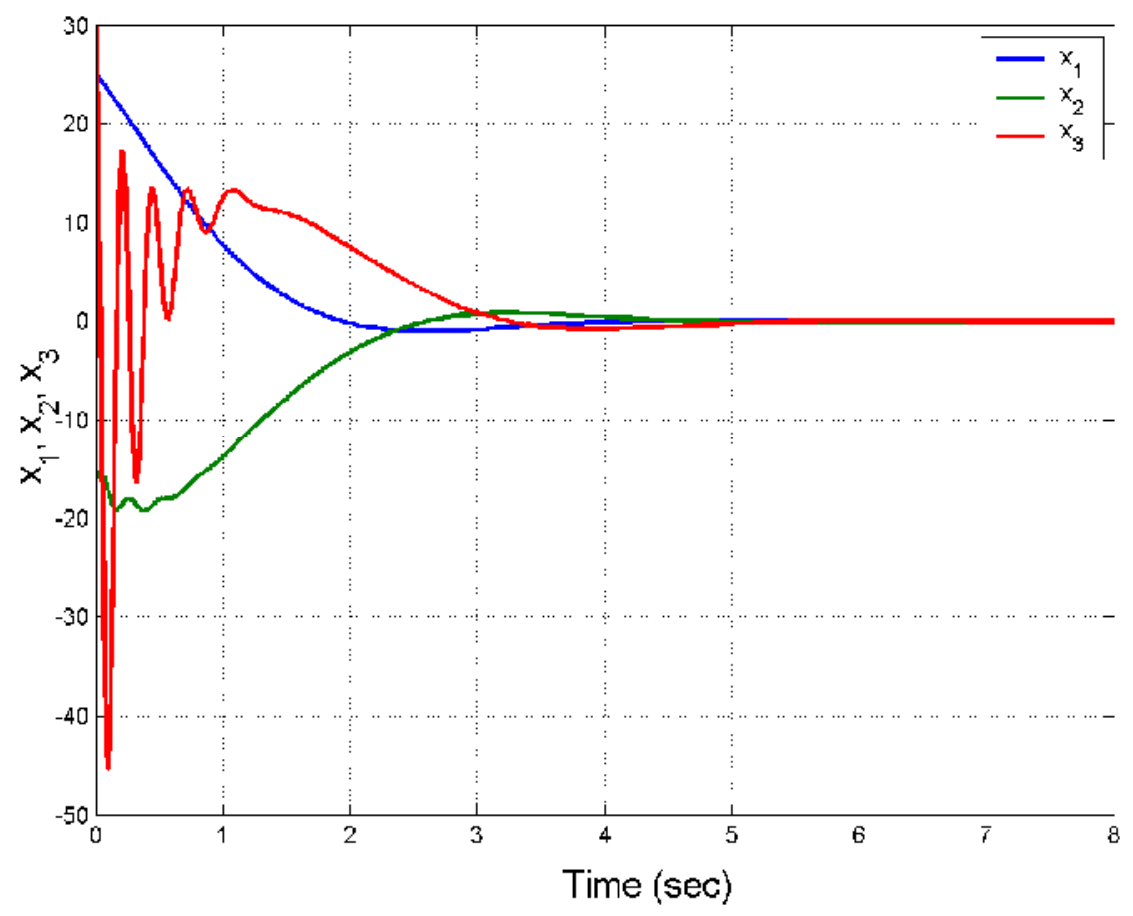

Figure 2. Time Responses of the Controlled Arneodo Chaotic System

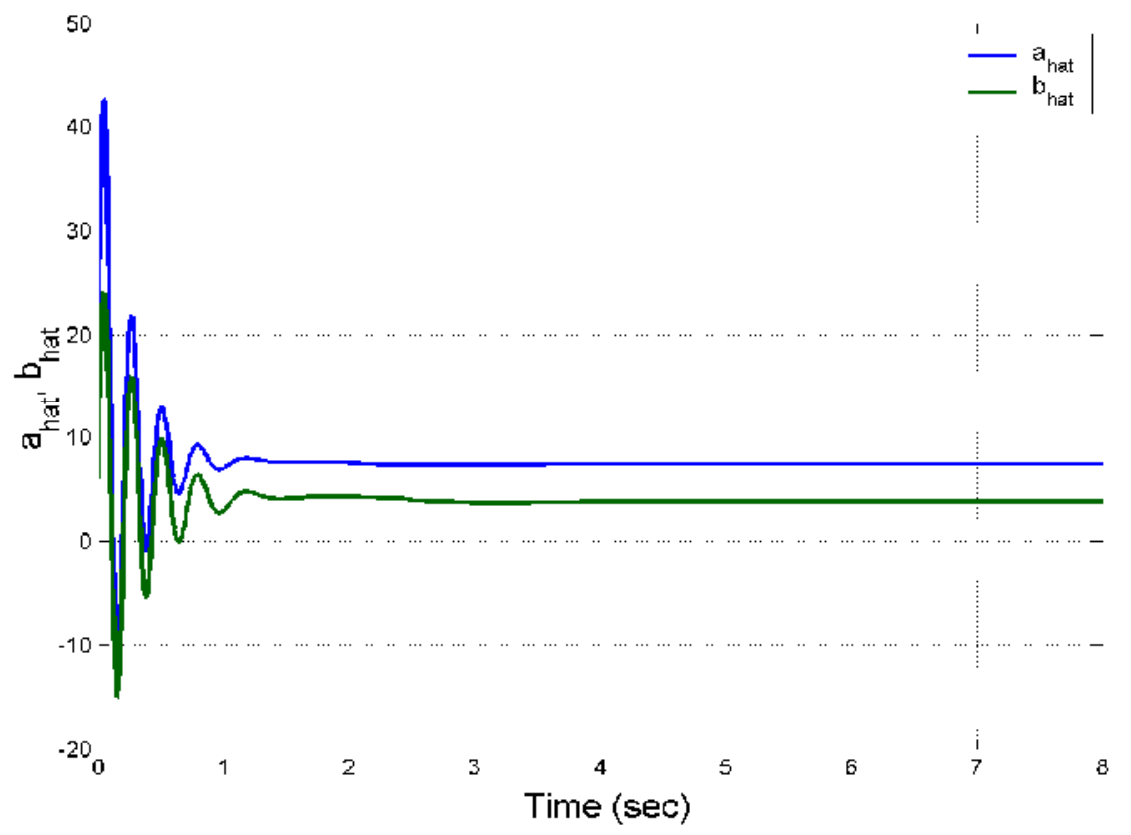

Figure 3. Time-History of the Parameter Estimates $\hat{a}(t), \hat{b}(t)$ 


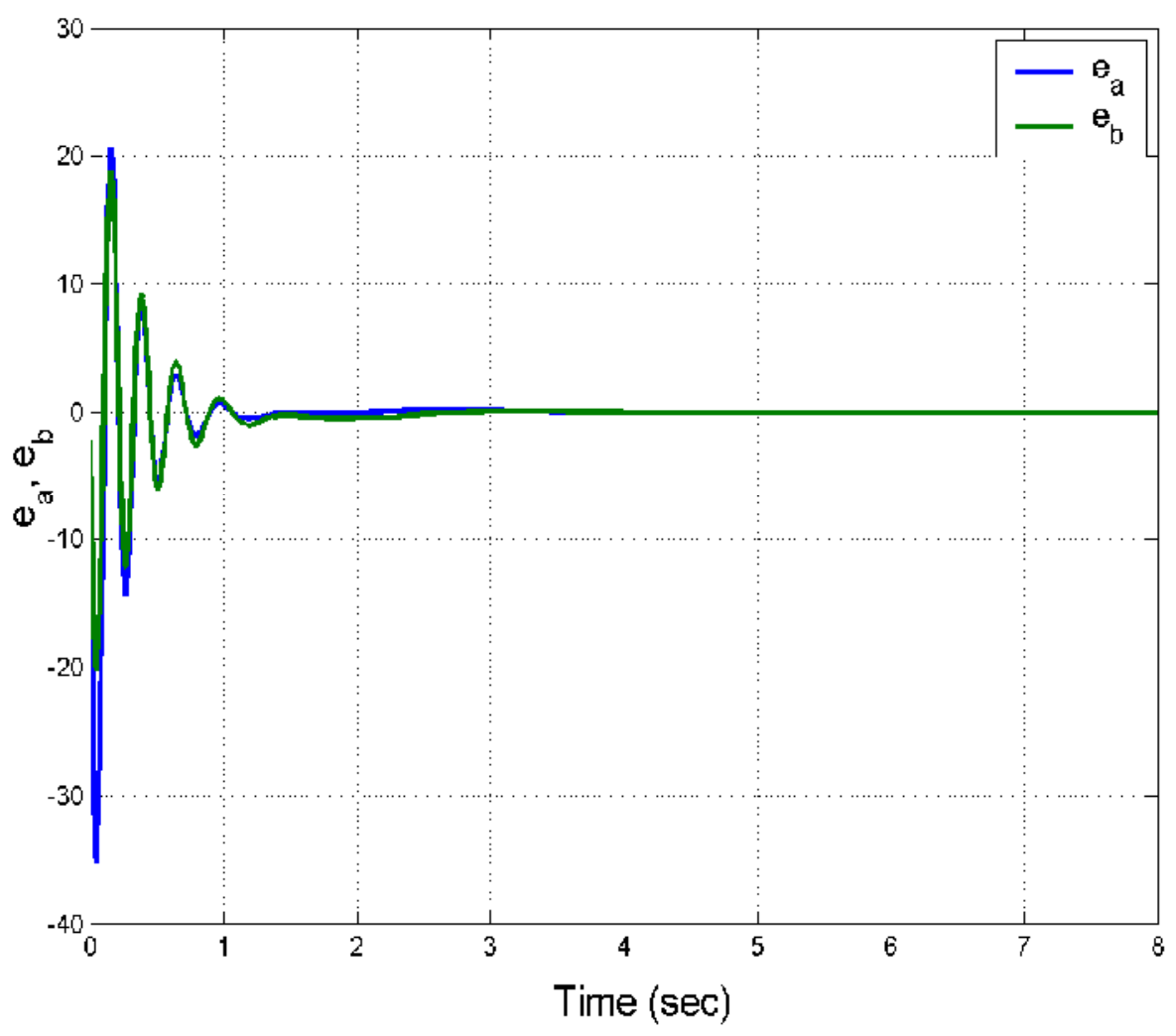

Figure 4. Time-History of the Parameter Estimation Error $e_{a}, e_{b}$

\section{Adaptive Backstepping Synchronization of Identical Arneodo CHAOTIC SySTEMS}

\subsection{Main Results}

In this section, we derive new results for the adaptive backstepping synchronization of identical Arneodo systems (1980) with unknown parameters.

As the master system, we take the Arneodo dynamics described by

$$
\begin{aligned}
& \dot{x}_{1}=x_{2} \\
& \dot{x}_{2}=x_{3} \\
& \dot{x}_{3}=a x_{1}-b x_{2}-x_{3}-x_{1}^{2}
\end{aligned}
$$

where $x_{i},(i=1,2,3)$ are the state variables and $a, b$ are unknown system parameters.

The system (16) is chaotic when the parameter values are taken as

$$
a=7.5, \quad b=3.8
$$


As the slave system, we consider the controlled Arneodo dynamics described by

$$
\begin{aligned}
& \dot{y}_{1}=y_{2} \\
& \dot{y}_{2}=y_{3} \\
& \dot{y}_{3}=a y_{1}-b y_{2}-y_{3}-y_{1}^{2}+u
\end{aligned}
$$

where $y_{i},(i=1,2,3,4)$ are the state variables and $u_{i},(i=1,2,3,4)$ are the nonlinear controllers to be designed.

The synchronization error is defined by

$e_{i}=y_{i}-x_{i}, \quad(i=1,2,3,4)$

Then the error dynamics is obtained as

$$
\begin{aligned}
& \dot{e}_{1}=e_{2} \\
& \dot{e}_{2}=e_{3} \\
& \dot{e}_{3}=a e_{1}-b e_{2}-e_{3}-\left(y_{1}+x_{1}\right) e_{1}+u
\end{aligned}
$$

We use backstepping control method to find an adaptive synchronizer $u(t)$ which uses the states of the master and slave systems and also the estimates $\hat{a}(t), \hat{b}(t)$ of the unknown parameters $a, b$.

We define parameter estimation errors as

$$
\begin{aligned}
& e_{a}(t)=a-\hat{a}(t) \\
& e_{b}(t)=b-\hat{b}(t)
\end{aligned}
$$

Note that

$$
\begin{aligned}
& \dot{e}_{a}(t)=-\dot{\hat{a}}(t) \\
& \dot{e}_{b}(t)=-\dot{\hat{b}}(t)
\end{aligned}
$$

The main result for the adaptive backstepping synchronizer design for the Arneodo systems (16) and (17) is described by the following theorem.

Theorem 2. The identical Arneodo chaotic systems (16) and (17) with unknown parameters and is globally and exponentially stabilized for all values of $x(0), y(0) \in R^{3}$ by the backstepping controller

$$
u(t)=-\left(\hat{a}+3-y_{1}-x_{1}\right) e_{1}-(5-\hat{b}) e_{2}-2 e_{3}
$$


where $\hat{a}(t), \hat{b}(t)$ are estimates of the unknown parameters $a, b$ and the parameter update law is given by

$\dot{\hat{a}}=\left(2 e_{1}+2 e_{2}+e_{3}\right) e_{1}+k_{a} e_{a}$

$\dot{\hat{b}}=-\left(2 e_{1}+2 e_{2}+e_{3}\right) e_{2}+k_{b} e_{b}$

with gains $k_{a}, k_{b}>0$.

Proof. We establish the main result using Lyapunov stability theory [48].

First, we define a Lyapunov function candidate

$V_{1}=\frac{1}{2} z_{1}^{2}$

where $z_{1}=e_{1}$.

Differentiating $V_{1}$ along the solutions of the error system (19), we get

$\dot{V}_{1}=z_{1} \dot{z}_{1}=e_{1} e_{2}=-e_{1}^{2}+e_{1}\left(e_{1}+e_{2}\right)$

Secondly, we choose the second Lyapunov function candidate as

$V_{2}=V_{1}+\frac{1}{2} z_{2}^{2}=\frac{1}{2}\left(z_{1}^{2}+z_{2}^{2}\right)$

where $z_{2}=e_{1}+e_{2}$.

Differentiating $V_{2}$ along the solutions of the error system (19), we get

$\dot{V}_{2}=-z_{1}^{2}-z_{2}^{2}+z_{2}\left(2 e_{1}+2 e_{2}+e_{3}\right)$

Finally, for the systems (19) and (21), we consider the Lyapunov function candidate

$V=V_{2}+\frac{1}{2} z_{3}^{2}+\frac{1}{2} e_{a}^{2}+\frac{1}{2} e_{b}^{2}=\frac{1}{2}\left(z_{1}^{2}+z_{2}^{2}+z_{3}^{2}+e_{a}^{2}+e_{b}^{2}\right)$

where $z_{3}=2 e_{1}+2 e_{2}+e_{3}$.

Differentiating $V$ along the solutions of the systems (19) and (21), we obtain

$\dot{V}=-z_{1}^{2}-z_{2}^{2}-z_{3}^{2}+z_{3}\left[\left(a+3-y_{1}-x_{1}\right) e_{1}+(5-b) e_{2}+2 e_{3}+u\right]-e_{a} \dot{\hat{a}}-e_{b} \dot{\hat{b}}$

Substituting the backstepping controller (22) into (29), we obtain

$\dot{V}=-z_{1}^{2}-z_{2}^{2}-z_{3}^{2}+z_{3}\left(e_{a} e_{1}-e_{b} e_{2}\right)-e_{a} \dot{\hat{a}}-e_{b} \dot{\hat{b}}$ 
That is,

$\dot{V}=-z_{1}^{2}-z_{2}^{2}-z_{3}^{2}+e_{a}\left(z_{3} e_{1}-\dot{\hat{a}}\right)+e_{b}\left(-z_{3} e_{2}-\dot{\hat{b}}\right)$

Substituting the parameter update law (23) into (30), we get

$\dot{V}=-z_{1}^{2}-z_{2}^{2}-z_{3}^{2}-k_{a} e_{a}^{2}-k_{b} e_{b}^{2}$

which is a negative definite function.

Hence, by Lyapunov stability theory [48], the proof is complete.

\subsection{Numerical Results}

For the numerical simulations, the fourth order Runge-Kutta method is used to solve the two systems of differential equations (16) and (17) with the adaptive control law (22) and the parameter update law (23).

We take the parameter values as in the chaotic case, viz.

$$
a=7.5, \quad b=3.8
$$

We take the positive gains as $k_{a}=6$ and $k_{b}=6$.

Suppose that the initial values of the estimated parameters are

$$
\hat{a}(0)=2, \hat{b}(0)=5
$$

We take the initial values of the master system (16) as

$$
x_{1}(0)=3, \quad x_{2}(0)=8, \quad x_{3}(0)=-1
$$

We take the initial values of the slave system (17) as

$$
y_{1}(0)=5, \quad y_{2}(0)=-10, \quad y_{3}(0)=9
$$

Figure 5 shows the chaos synchronization of the identical Arneodo systems.

Figure 6 shows the time-history of the synchronization error $e_{1}, e_{2}, e_{3}$.

Figure 7 shows the time-history of the parameter estimates $\hat{a}(t), \hat{b}(t)$.

From this figure, it is clear that the parameter estimates converge to the original values $a=7.5$ and $b=3.8$, respectively.

Figure 8 shows the time-history of the parameter estimation errors $e_{a}, e_{b}$. 

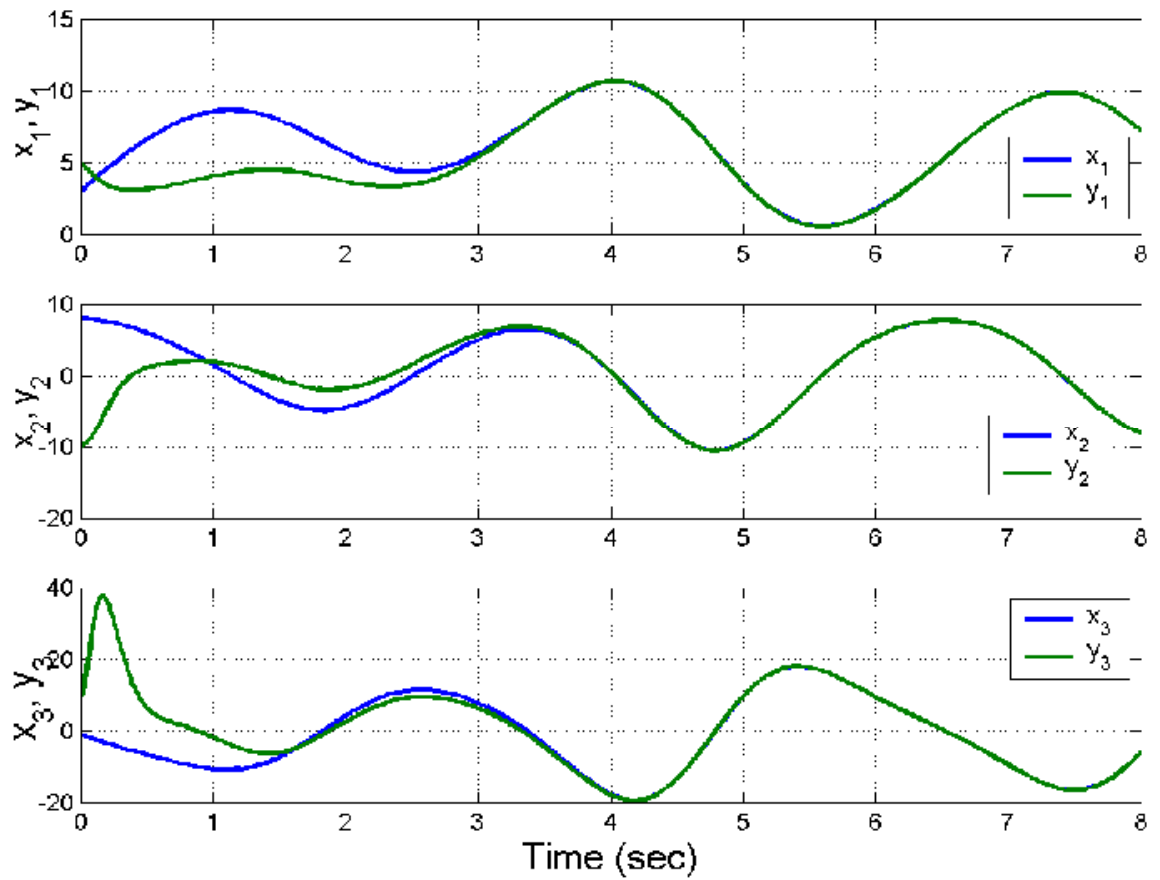

Figure 5. Adaptive Synchronization of the Arneodo Systems

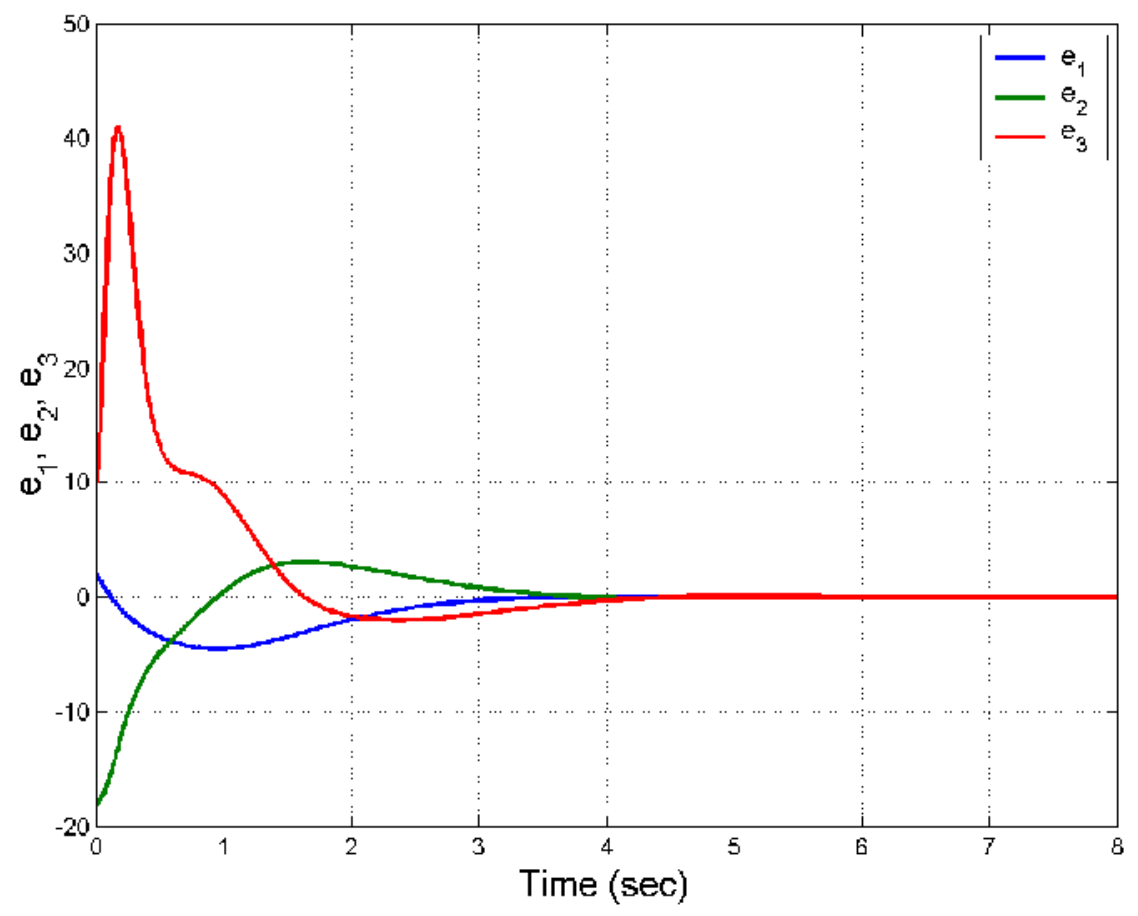

Figure 6. Time-History of the Synchronization Error $e_{1}, e_{2}, e_{3}$ 


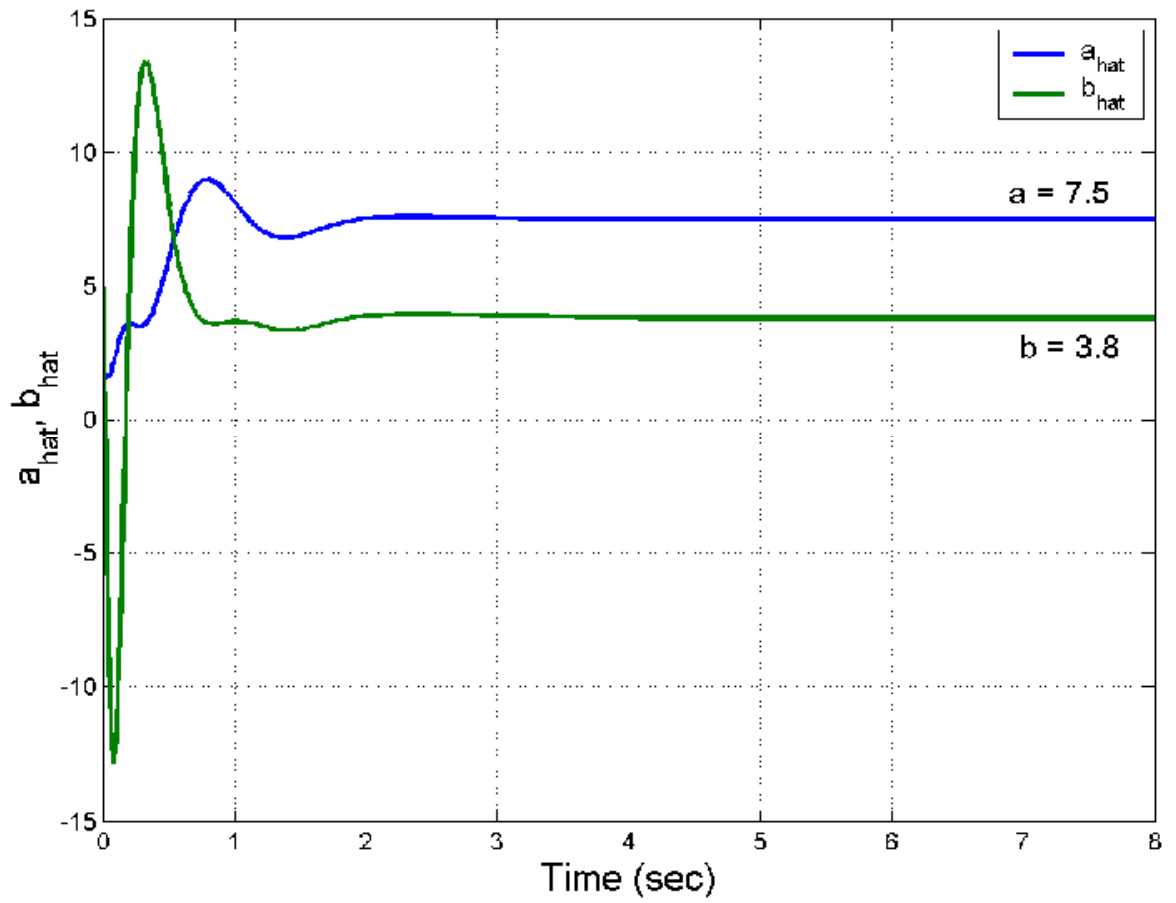

Figure 7. Time-History of the Parameter Estimates $\hat{a}(t), \hat{b}(t)$

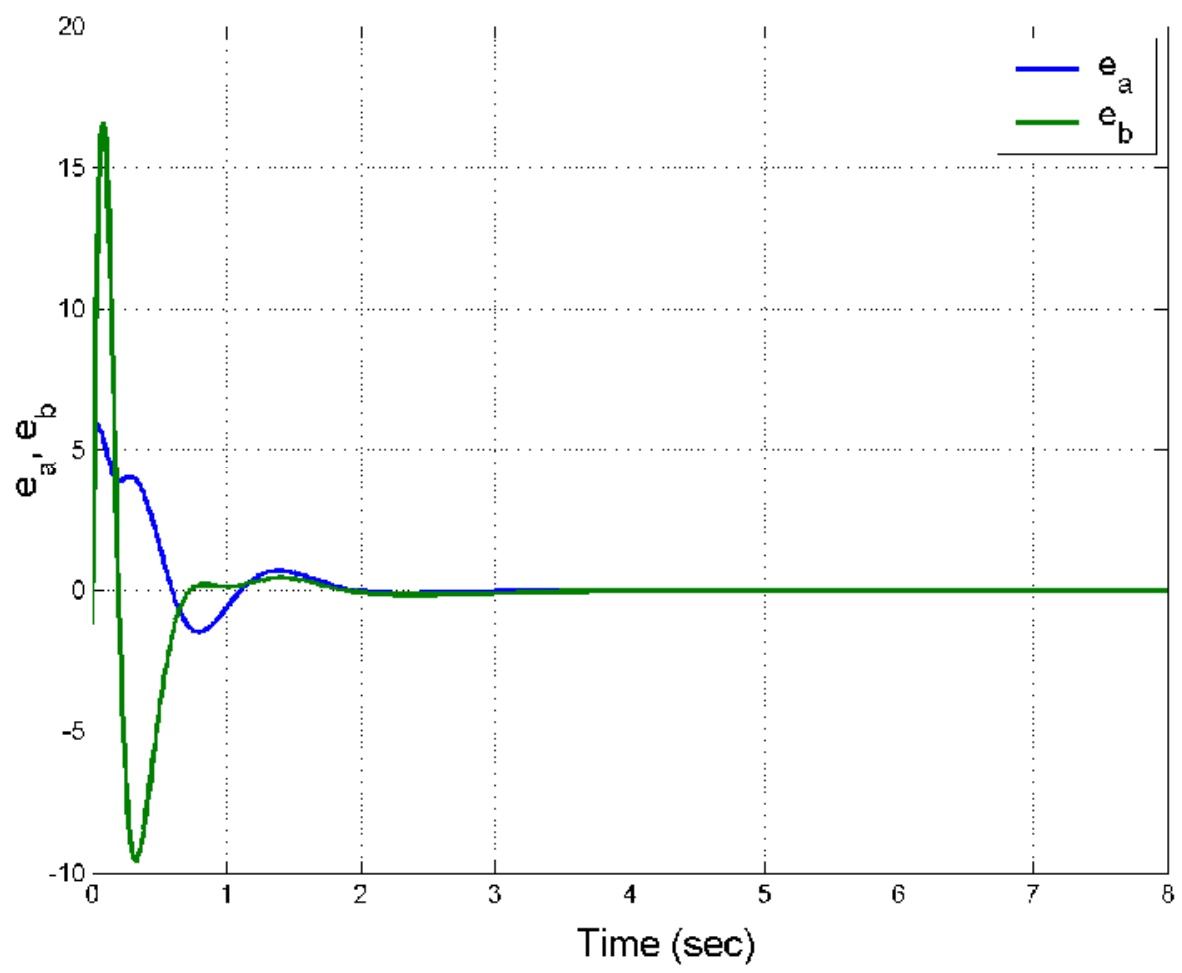

Figure 8. Time-History of the Parameter Estimation Error $e_{a}, e_{b}$ 


\section{Conclusions}

In this paper, we have applied backstepping control method to derive new results for the adaptive stabilization and synchronization of the Arneodo system (1980) with unknown system parameters. First, an adaptive controller law was designed via backstepping control method for stabilizing the Arneodo system (1980) to its unstable equilibrium at the origin. Next, an adaptive synchronizer law was designed via backstepping control method for synchronizing identical Arneodo systems. The main results derived in this paper were proved using Lyapunov stability theory. Numerical simulations using MATLAB have been provided to validate and demonstrate the effectiveness of the proposed adaptive backstepping control and backstepping synchronization schemes for the Arneodo chaotic system.

\section{REFERENCES}

[1] Lorenz, E.N. (1963) "Deterministic nonperiodic flow," Journal of the Atmospheric Sciences, Vol. 20, pp 130-141.

[2] Rössler, O.E. (1976) “An equation for continuous chaos,” Physics Letters, Vol. 57A, No. 5, pp $397-$ 398.

[3] Leipnik, R.B. \& Newton, T.A. (1981) "Double strange attractors in rigid body motion," Physics Letters A, Vol. 86, pp 63-67.

[4] Chen, G. \& Ueta, T. (1999) "Yet another chaotic attractor," International J. Bifurcation and Chaos, Vol. 9, pp 1465-1466.

[5] Lü, J. \& Chen, G. (2002) "A new chaotic attractor coined," International J. Bifurcation and Chaos, Vol. 12, pp 659-661.

[6] Wang, X., Tian, L. \& Yu, L. (2006) "Adaptive control and slow manifold analysis of a new chaotic system,” Internat. J. Nonlinear Science, Vol. 21, pp 43-49.

[7] Sun, M., Tian, L., Jiang, S. \& Xun, J. (2007) "Feedback control and adaptive control of the energy resource chaotic system," Chaos, Solitons \& Fractals, Vol. 32, pp 168-180.

[8] Ott, E., Grebogi, C. \& Yorke, J.A. (1990) “Controlling chaos”, Phys. Rev. Lett., Vol. 64, pp 11961199.

[9] Richter, H. (2002) "Controlling chaotic system with multiple strange attractors," Physics Letters A, Vol. 300, pp 182-188.

[10] Ge, S.S., Wang, C. \& Lee, T.H. (2000) "Adaptive backstepping control of a class of chaotic systems," International Journal of Bifurcation and Chaos, Vol. 10, pp 1149-1156.

[11] Feki, M. (2003) "An adaptive feedback control of linearizable chaotic systems," Chaos, Solitons \& Fractals, Vol. 15, No. 5, pp 883-890.

[12] Yassen, M.T. (2003) "Adaptive control and synchronization of a modified Chua's circuit system," Applied Mathematics and Computation, Vol. 135, No. 1, pp 113-128.

[13] Pecora, L.M. \& Carroll, T.L. (1990) "Synchronization in chaotic systems", Phys. Rev. Lett., Vol. 64, pp 821-824.

[14] Lakshmanan, M. \& Murali, K. (1996) Nonlinear Oscillators: Controlling and Synchronization, World Scientific, Singapore.

[15] Han, S.K., Kerrer, C. \& Kuramoto, Y. (1995) "Dephasing and bursting in coupled neural oscillators", Phys. Rev. Lett., Vol. 75, pp 3190-3193.

[16] Petrov, V., Gaspar, V,. Masere, J. \& Showalter, K. (1993) "Controlling chaos in the BelousovZhabotinsky reaction," Nature, Vol. 361, pp 240-243.

[17] Blasius, B., Huppert, A. \& Stone, L. (1999) "Complex dynamics and phase synchronization in spatially extended ecological system", Nature, Vol. 399, pp 354-359.

[18] Skinner, J. E. (1994) "Low-dimensional chaos in biological systems," Nature Biotechnology, Vol. 12, pp 596-600.

[19] Garfinkel, A., Spano, M. L., Ditto, W. L. \& Weiss, J. A. (1992) “Controlling cardiac chaos,” Science, Vol. 257, pp 1230-1235.

[20] Yang, X.S. \& Yuan, Q. (2005) "Chaos and transient chaos in simple Hopfield neural networks," Neurocomputing, Vol. 69, No. 1-3, pp 232-241. 
[21] Nakamura, Y. \& Sekiguchi, A. (2001) "Chaotic mobile robot", IEEE Transactions on Robotics and Automation, Vol. 17, pp 898-904.

[22] Volos, C.K., Kyrpianidisb, I.M. \& Stouboulosb, I.N. (2012) "A chaotic path planning generator for autonomous mobile robots," Robotics and Autonomous Systems, Vol. 60, No. 4, pp 651-656.

[23] Feki, M. (2003) "An adaptive chaos synchronization scheme applied to secure communication", Chaos, Solitons and Fractals, Vol. 18, pp 141-148.

[24] Murali, K. \& Lakshmanan, M. (1998) "Secure communication using a compound signal from generalized synchronizable chaotic systems", Phys. Rev. Lett. A, Vol. 241, pp 303-310.

[25] Ho, M.C. \& Hung, Y.C. (2002) "Synchronization of two different chaotic systems by using generalized active control”, Physics Letters A, Vol. 301, pp 424-428.

[26] Huang, L., Feng, R. \& Wang, M. (2004) "Synchronization of chaotic systems via nonlinear control", Physics Letters A, Vol. 320, pp 271-275.

[27] Lei, Y., Xu, W. \& Zheng, H. (2005) "Synchronization of two chaotic nonlinear gyros using active control," Physics Letters A, Vol. 343, No. 1, pp 153-158.

[28] Tang, R.A., Liu, Y.L. \& Xue, J.K. (2009) "An extended active control for chaos synchronization," Physics Letters A, Vol. 373, No. 6, pp 1449-1454.

[29] Sundarapandian, V. (2011) "Global chaos synchronization of Lorenz and Pehlivan chaotic systems by nonlinear control", International Journal of Advances in Science and Technology, Vol. 2, No. 3, pp 19-28.

[30] Sundarapandian, V. (2012) "Global chaos synchronization of Sprott L and M systems by active control," International J. Control Theory and Computer Modelling, Vol. 2, No. 4, pp 21-35.

[31] Park, J.H. (2007) “Adaptive controller design for modified projective synchronization of GenesioTesi chaotic system with uncertain parameters”, Chaos, Solitons \& Fractals, Vol. 34, pp 1154-1159.

[32] Park, J.H., Lee, S.M. \& Kwon, O.M. (2007) "Adaptive synchronization of Genesio-Tesi chaotic system via a novel feedback control," Physics Letters A, Vol. 371, pp 263-270.

[33] Sundarapandian, V. (2011) "Adaptive control and synchronization of hyperchaotic Chen system", International Journal of Information Technology Convergence and Services, Vol. 1, No. 3, pp 22-33.

[34] Sundarapandian, V. (2011) "Adaptive synchronization of hyperchaotic Lorenz and hyperchaotic Lü systems", International Journal of Instrumentation and Control Systems, Vol. 1, pp 1-18.

[35] Sundarapandian, V. (2011) "Adaptive control and synchronization of hyperchaotic Cai system", International Journal of Control Theory and Computer Modeling, Vol. 1, No. 1, pp 1-13.

[36] Park, J.H. \& Kwon, O.M. (2003) "A novel criterion for delayed feedback control of time-delay chaotic systems", Chaos, Solitons and Fractals, Vol. 17, pp 709-716.

[37] Zhao, J. \& Lü, J. (2006) "Using sampled-data feedback control and linear feedback synchronization in a new hyperchaotic system," Chaos, Solitons \& Fractals, Vol. 35, pp. 376-382.

[38] Konishi, K., Hirai, M. \& Kokame, H. (1998) "Sliding mode control for a class of chaotic systems", Physics Letters A, Vol. 245, pp 511-517.

[39] Sundarapandian, V. (2011) "Hybrid chaos synchronization of hyperchaotic Newton-Leipnik systems by sliding mode control”, International Journal of Control Theory \& Computer Modelling, Vol. 1, No.2, pp 1-10.

[40] Sundarapandian, V. (2011) "Global chaos synchronization of Pehlivan systems by sliding mode control”, International Journal of Computer Science \& Engineering, Vol. 3, 2163-2169.

[41] Sundarapandian, V. (2011) "Sliding mode controller design for synchronization of Shimizu-Morioka chaotic system", International Journal of Information Sciences and Techniques, Vol. 1, No. 1, pp 20 29.

[42] Sundarapandian, V. (2011) "Global chaos synchronization of four-wing chaotic systems by sliding mode control", International Journal of Control Theory and Computer Modeling, Vol., 1 No. 1, pp 1531.

[43] Yu, Y.G. \& Zhang, S.C. (2006) "Adaptive backstepping synchronization of uncertain chaotic systems", Chaos, Solitons and Fractals, Vol. 27, pp 1369-1375.

[44] Cai, G. \& Tu, W. (2007) “Adaptive Backstepping Control of the Uncertain Unified Chaotic System”, International Journal of Nonlinear Science, Vol. 4, pp 17-24.

[45] Suresh, R. \& Sundarapandian, V. (2012) "Global chaos synchronization of Windmi and Coullet chaotic systems by backstepping control”, Far East Journal of Mathematical Sciences, Vol. 67, No. 2, pp 265-282.

[46] Suresh, R. \& Sundarapandian, V. (2012) "Hybrid synchronization of n-scroll Chua and Lur'e chaotic systems via backstepping control with novel feedback," Archives of Control Sciences, Vol. 22, No. 3, pp 255-278. 
[47] Arneodo, A., Coullet, P. \& Tresser, C. (1980) "Occurrence of strange attractors in three-dimensional Volterra equations,” Physics Letters A, Vol. 79, pp 259-263.

[48] Hahn, W. (1967) The Stability of Motion, Springer, New York.

\section{Author}

Dr. V. Sundarapandian earned his Doctor of Science degree in Electrical and Systems Engineering from Washington University, St. Louis, USA in May 1996. He is Professor and Dean at the R \& D Centre at Vel Tech Dr. RR \& Dr. SR Technical University, Chennai, Tamil Nadu, India. He has published over 290 refereed papers in international journals. He has published over 180 papers in National and International Conferences. $\mathrm{He}$ is an Indian Chair of AIRCC. He is the Editor-in-Chief of the AIRCC Journals IJICS, IJCTCM, IJITCA, IJCCMS and IJITMC. He has recently authored a text-book titled "Ordinary and Partial Differential Equations" (2012) published by McGraw Hill Education, India. His research interests are Linear and Nonlinear Control Systems,

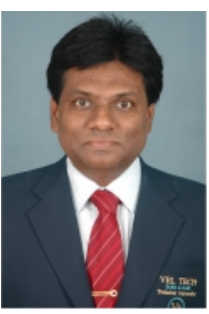
Chaos Theory and Control, Soft Computing, Optimal Control, Operations Research, Mathematical Modelling and Scientific Computing. He has delivered several Key Note Lectures on Control Systems, Chaos Theory and Control, Operations Research, Mathematical Modelling, and Scientific Computing using MATLAB and SCILAB. 